\title{
jsMolEditor: an open source molecule editor for the next generation web
}

\author{
L Duan \\ From 5th German Conference on Cheminformatics: 23. CIC-Workshop \\ Goslar, Germany. 8-10 November 2009
}

Molecule editor have become essential building blocks for modern chemistry related databases with structural operation available. It was quite a challenging task to input molecule structures in a web browser before Java Applets technology was applied in chemoinformatics. Until now, Java Applets are still the de facto standard for web based databases. With the development of web technologies and script programming, Ajax, a Web 2.0 technology, is becoming mainstream in web development. This gradually eliminates the need for plug-ins such as Java and ActiveX. Therefore, it is possible to develop powerful web-based tools without plug-ins.

This poster introduces a JavaScript based molecule editor: jsMolEditor, which had made a significant difference from other editors available on web pages. Unlike server side editors such as PubChem editor [1], jsMolEditor implemented all its functionality in JavaScript on the client side. Thus it is not necessary to maintain a connection to a server during operation. jsMolEditor works completely independently on the client side. The JavaScript nature of jsMolEditor also made it able to run in standard web browsers without specific plugins or virtual machines. The $2 \mathrm{D}$ drawing system deals with web browser differences. By combining Canvas and VML, jsMolEditor was able to work on all common web browsers including Internet Explorer, Firefox, Safari, Opera and Chrome. Instead of building jsMolEditor in JavaScript from scratch, GWT [2] is utilized as a crosscompiler from Java to JavaScript, which enables the reuse of existing Java chemoinformatics frameworks. jsMolEditor used a ported version of MX [3] as the bottom layer framework to handle basic molfile I/O. Thanks to MX's open source license, the development of jsMolEditor saves a lot of time by eliminating the process of "reinventing the wheel". The poster

School of Pharmacy, East China University of Science and Technology, 130 Meilong Road, Shanghai 200237, PR China represents not only jsMolEditor itself, but also the building process of jsMolEditor and the feasibility of applying the same concept to other chemistry gadgets on web pages, such as spectrum viewers.

jsMolEditor s source code is available at http://github. com/chemhack/jsmoleditor/.

Published: 4 May 2010

References

1. PubChem Server Side Structure Editor. [http://pubchem.ncbi.nlm.nih.gov/ edit/].

2. GWT, Google Web Toolkit. [http://code.google.com/webtoolkit/].

3. MX Chemoinformatics Toolkit. [http://metamolecular.com/mx].

doi:10.1186/1758-2946-2-S1-P3

Cite this article as: Duan: jsMolEditor: an open source molecule editor for the next generation web. Journal of Cheminformatics 2010 2(Suppl 1):P3.

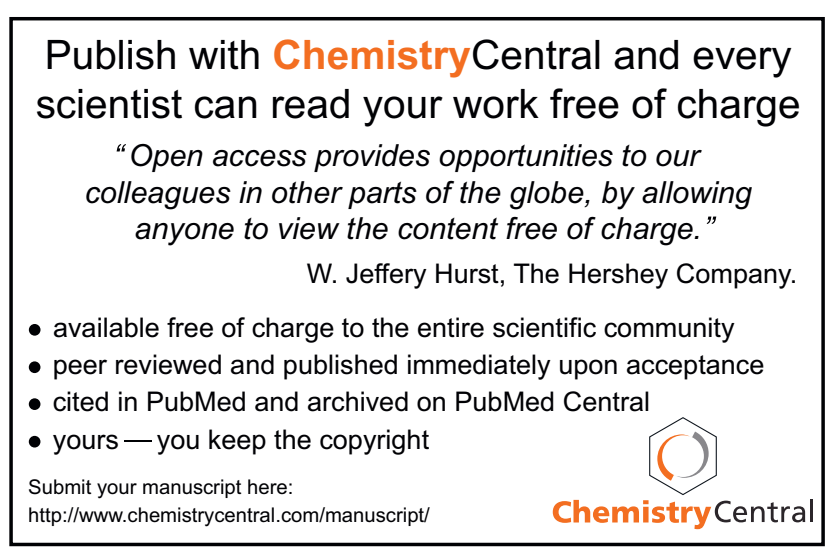

